Methods Secondary analysis of the Edinburgh pneumonia database, a prospective observational study of CAP (2005-2010). All discharged patients were included. Follow-up data were obtained from a database linked to national morbidity and mortality registers. Outcomes were assessed using cox proportional hazards regression adjusting for confounding variables (age, gender, previous cardiovascular events, ACE-inhibitor/anti-platelet use, smoking status and severity of pneumonia).

Results Data from 1631 patients with complete follow-up were analysed. Readmissions occurred in 728 patients (44.6\%) with 157 readmissions within 30 days of discharge. 133 patients had a further hospitalisation with CAP. The 1 year mortality rate was $12.8 \%$.

523 patients were current statin users. There were significant differences between statin and non-statin users. Statin users were older, suffering more cardiovascular disease, stroke, diabetes, renal disease, COPD and a greater severity of pneumonia.

1 year mortality rates were similar in statin and non-statin users. After adjusting for baseline differences, statins were associated with a non-significant trend towards lower 1 year mortality HR $0.78(0.55-1.1)$. When adjusted for the propensity score, the difference in mortality became statistically significant HR 0.70 (0.50-0.98).

In the fully adjusted analysis, statins were not significantly associated with readmissions HR $0.85(0.70-1.03)$ but were associated with a significantly lower risk of recurrent pneumonia HR $0.60(0.37-0.96)$. There was no association with reduced cardiovascular hospitalisations HR 0.94(0.65-1.34). There were no beneficial effects seen with either antiplatelet or ACE-inhibitor use following CAP.

Conclusion Statins are associated with reduced 1 year mortality and a significantly lower rate of recurrent pneumonia. This is the first study to show that statins may improve long term outcomes in CAP, and that the associated morbidity and mortality can be modified.

\section{S123 INCREASED RISK OF UPPER RESPIRATORY INFECTION WITH ADDITION OF INTERMITTENT BOLUS-DOSE VITAMIN D SUPPLEMENTATION TO A DAILY LOW-DOSE REGIMEN}

${ }^{1} \mathrm{AR}$ Martineau, ${ }^{1} \mathrm{Y}$ Hanifa, ${ }^{1} \mathrm{RL}$ Hooper, ${ }^{1} \mathrm{KD}$ Witt, ${ }^{1} \mathrm{M}$ Patel, ${ }^{1} \mathrm{~A}$ Syed, ${ }^{1} \mathrm{DA}$ Jolliffe, ${ }^{1} \mathrm{PM}$ Timms, ${ }^{1} \mathrm{Z}$ Balayah, ${ }^{1} \mathrm{~N}$ Stevens, ${ }^{2} \mathrm{DA}$ Clark, ${ }^{1} \mathrm{~S}$ Eldridge, ${ }^{2} \mathrm{~N}$ Barnes, ${ }^{1} \mathrm{CJ}$ Griffiths; ${ }^{1}$ Queen Mary University of London, London, United Kingdom; ${ }^{2}$ Barts Health NHS Trust, London, United Kingdom

\subsection{6/thoraxjnl-2013-204457.130}

Introduction and Objectives Meta-analysis of clinical trials of vitamin $\mathrm{D}$ supplementation for the prevention of acute respiratory infection (ARI) shows a protective effect in the general population, but there is controversy regarding the optimal dosing regimen. Low-dose vitamin D supplementation is already recommended in older adults for prevention of fractures and falls, but clinical trials investigating whether higher doses could provide additional protection against ARI are lacking.

Methods We conducted a double-blind cluster-randomised placebo-controlled trial of high- vs. low-dose vitamin D supplementation in residents and staff of sheltered accommodation schemes in London, UK. 108 schemes were allocated to receive the intervention (vitamin $\mathrm{D}_{3} 2.4 \mathrm{mg}$ 2-monthly $+10 \mu \mathrm{g}$ daily for residents; $3 \mathrm{mg}$ 2-monthly for staff) or control (vitamin $\mathrm{D}_{3} 10 \mu \mathrm{g}$ daily for residents, nil for staff) over the course of one year. The primary endpoint of the trial was time from first dose of study medication to date of first ARI, determined by a validated acute respiratory symptom score recorded prospectively in a symptom diary. Secondary outcomes included time to first upper / lower respiratory infections (URI/LRI) and mean serum 25-hydroxyvitamin D $(25[\mathrm{OH}] \mathrm{D})$ concentration.

Results 240 participants were included in the intention-to-treat analysis (137 participants in 54 schemes allocated to intervention, mean baseline $25[\mathrm{OH}] \mathrm{D} 43.8 \mathrm{nmol} / \mathrm{L}$ vs. 103 participants in 54 schemes allocated to control, mean baseline $25[\mathrm{OH}] \mathrm{D}$ $43.8 \mathrm{nmol} / \mathrm{L}$ ). Median time to ARI was 203 days in the intervention arm and 227 days in the control arm (adjusted HR 1.18, $95 \%$ CI 0.80 to $1.74, \mathrm{p}=0.42$ ). Allocation to the intervention arm of the trial was associated with increased risk of URI (adjusted HR 1.48, 95\% CI 1.02 to $2.16, \mathrm{p}=0.04$ ), but not with altered risk of LRI (adjusted HR 1.12, 95\% CI 0.73 to $1.70, \mathrm{p}=0.61)$. Mean serum 25(OH)D concentration at 1 year was $84.8 \mathrm{nmol} / \mathrm{L}$ vs. $58.5 \mathrm{nmol} / \mathrm{L}$ in intervention vs. control arms $(\mathrm{p}<0.0001)$.

Conclusions Addition of intermittent bolus-dose vitamin D supplementation to a daily low-dose regimen improved vitamin $\mathrm{D}$ status in older adults and their carers, but it did not influence risk of ARI, and was less effective at preventing URI.

\section{S124 DERIVATION AND VALIDATION OF THE BRONCHIECTASIS SEVERITY INDEX: AN INTERNATIONAL MULTICENTRE OBSERVATIONAL STUDY}

${ }^{1} \mathrm{JD}$ Chalmers, ${ }^{2} \mathrm{P}$ Goeminne, ${ }^{1} \mathrm{~S}$ Aliberti, ${ }^{3} \mathrm{M} M \mathrm{MCD}^{2}$ nell, ${ }^{4} \mathrm{~S}$ Lonzi, ${ }^{3} \mathrm{~J}$ Davidson, ${ }^{1} \mathrm{~L}$ Poppelwell, ${ }^{1} \mathrm{~W}$ Salih, ${ }^{4} \mathrm{~A}$ Pesci, ${ }^{2} \mathrm{~L}$ Dupont, ${ }^{1} \mathrm{TC}$ Fardon, ${ }^{3} \mathrm{~A}$ De Soyza, ${ }^{5} \mathrm{AT}$ Hill; ${ }^{7}$ University of Dundee, Dundee, UK; ${ }^{2}$ University Hospital Gasthuisberg, Leuven, Belgium; ${ }^{3}$ University of Newcastle, Newcastle, UK; ${ }^{4}$ University of Milan Bicocca, Monza, Italy; ${ }^{5}$ Royal Infirmary of Edinburgh, Edinburgh, UK

\subsection{6/thoraxjnl-2013-204457.131}

Introduction There are no risk stratification tools for morbidity and mortality in bronchiectasis. As more treatments become available, it is important to identify patients at risk of exacerbations, hospital admissions and mortality to target novel therapies.

Methods A prospective observational study at a specialist bronchiectasis clinic in Edinburgh, UK was used to derive a bronchiectasis severity index using cox-proportional hazards regression to identify independent predictors of mortality and hospital admission over 4 years follow-up. Averaged ß-coefficients were used to award points for each independent variable and the discrimination of a derived score was tested using the area under the receiver operator characteristic curve (AUC). The score was validated in independent cohorts from Dundee, UK $(\mathrm{N}=218)$, Leuven, Belgium ( $\mathrm{N}=253)$, Monza, Italy $(\mathrm{N}=105)$ and Newcastle, UK $(\mathrm{N}=126)$.

Results 608 patients were included in the derivation cohort. Independent predictors of future hospital admissions were prior hospital admissions hazard ratio (HR) 13.5 (9.40-19.46), MRC dyspnoea score $>4$, HR 2.42 (1.66-3.52), FEV $_{1}<30 \%$ predicted HR 1.52 (1.03-2.25), Pseudomonas aeruginosa colonisation HR 2.16 (1.36-3.43), colonisation with other organisms HR 1.66 (1.12-2.44) and > 3 lobes involved on HRCT HR $1.48(1.02-2.15)$. In the model for mortality, independent predictors were Age $>70$ years 8.57 (1.15-63.63), $\mathrm{FEV}_{1}<30 \%$ predicted HR 4.47 (1.60-12.53), prior hospital admissions HR $2.43(1.30-4.53)$ and 3 or more exacerbations per year prior to the study HR 2.03 (1.02-4.03). 
The bronchiectasis severity index derived from these models was composed of prior hospitalisation (5 points), MRC dyspnoea score (0-3 points), $\mathrm{FEV}_{1}$ (0-3 points), bacterial colonisation (0- 3 points) Age (0-6 points) BMI $<18.5$ (2 points) Exacerbation frequency (0-2 points) and radiological extent (1 point). The AUC for mortality was $0.80(0.74-0.86)$ and the AUC for hospitalisation was $0.88(0.84-0.91)$. There was a clear difference in exacerbation frequency and quality of life using the St. Georges Respiratory Questionnaire between patients classified as low, intermediate and high risk by the score ( $\mathrm{p}<0.0001$ for all comparisons).

In the validation cohorts, the AUC for mortality ranged from 0.81-0.84 and for hospitalisation was AUC 0.80-0.88.

Conclusions The bronchiectasis severity index identifies patients at risk of future mortality, hospital admissions and exacerbations.

\section{S125 A RETROSPECTIVE STUDY CHARACTERISING CILIARY ULTRASTRUCTURE, LIGHT MICROSCOPY AND SPUTUM MICROBIOLOGY ASSOCIATIONS WITH LUNG FUNCTION DECLINE IN A LARGE ADULT PRIMARY CILIARY DYSKINESIA COHORT}

A Shah, A Rogers, A Shoemark, D Bilton, R Wilson, MR Loebinger; Royal Brompton \& Harefield NHS Foundation Trust, London, UK;

\subsection{6/thoraxjnl-2013-204457.132}

Background Primary ciliary dyskinesia (PCD) is an inherited disease related to ciliary dysfunction, with heterogeneity in clinical presentation, prognosis and ciliary ultrastructure. Our study aimed to comprehensively characterise a large cohort with respect to ciliary ultrastructure, beat frequency, sputum microbiology, mortality and lung function decline.

Method A cohort of 100 adult PCD patients was identified at a tertiary respiratory centre. A retrospective analysis of clinical age at presentation and diagnosis alongside ciliary ultrastructure, nasal nitric oxide, beat frequency, sputum microbiology, lung function at diagnosis and follow-up and mortality were recorded. Non-parametric multi-parameter analysis of variance and Spearman rank correlation statistical analysis was performed to identify significant associations with decline in lung function (FEV1\%/year). Median duration of follow-up was 7.5years (range 2-30years).

Results Overall mortality was 4\% (median age of death 55 years). $12 \%$ of patients had a central pair/transposition defect, $37 \%$ missing outer dynein arms, 15\% missing inner dynein arms, $28 \%$ no arms, and 3\% had normal ultrastructure. There was no significant correlation between ciliary ultrastructure, beat frequency (range $0-13.9 \mathrm{~Hz}$ ) and nasal nitric oxide with clinical age at presentation (range 1-26 years) and diagnosis (range 1-72 years) or lung function at presentation and decline with followup. There was additionally no significant association between sputum isolation including Pseudomonas aeruginosa with lung function decline. $44 \%$ of patients had Pseudomonas aeruginosa chronic infection. The incidence of NTM colonisation was low (4\%). Aspergillus species colonisation was additionally low (5\%). The average lung function decline in the cohort was $1.45 \%$ FEV1/year.

Conclusions Comprehensive characterisation of an adult PCD cohort with ciliary ultrastructure, light microscopy, clinical presentation and follow-up data shows a relatively favourable outcome with optimum care. Ciliary ultrastructure, beat frequency and nasal nitric oxide does not predict prognosis. Contrary to parallel diagnoses such as cystic fibrosis and adult idiopathic bronchiectasis, microbiological isolation of Pseudomonas aeruginosa is not associated with a more rapid decline in lung function with optimal prophylaxis and care. Contrary to recent suggestion of low ciliary beat frequency and low nasal nitric oxide association with NTM susceptibility, we did not find a high incidence of NTM or Aspergillus species within this cohort.

\section{S126 MOLECULAR EPIDEMIOLOGICAL ANALYSIS SUGGESTS CROSS INFECTION WITH PSEUDOMONAS AERUGINOSA IS RARE IN NON-CYSTIC FIBROSIS BRONCHIECTASIS}

${ }^{1} \mathrm{~A}$ De Soyza, ${ }^{2} \mathrm{~A}$ Perry, ${ }^{3} \mathrm{AJ}$ Hall, ${ }^{4} \mathrm{~S}$ Sunny, ${ }^{2} \mathrm{KE}$ Walton, ${ }^{5} \mathrm{~N}$ Mustafa, ${ }^{5} \mathrm{~J}$ Turton, ${ }^{5} \mathrm{DT}$ Kenna, ${ }^{3} \mathrm{C}$ Winstanley; ${ }^{1}$ ICM Newcastle University \& Freeman Hospital Bronchiectasis service, Newcastle, UK; ${ }^{2}$ Department of Medical Microbiology, Newcastle upon Tyne Hospitals Trust, Newcastle, UK; ${ }^{3}$ Institute of Infection and Global Health, University of Liverpool, Liverpool, UK; ${ }^{4}$ Sir William Leech Centre for Respiratory Research and Freeman Hospital Adult Bronchiectasis Unit, Newcastle upon Tyne Hospitals Trust, Newcastle, UK; ${ }^{5}$ Public Health England, Colindale, London, UK

\subsection{6/thoraxjnl-2013-204457.133}

Background Non Cystic Fibrosis Bronchiectasis (NCFBr) is a cause of significant morbidity and mortality. Pseudomonas aeruginosa, a key pathogen in $\mathrm{NCFBr}$, is associated with premature mortality. Globally, common clones of P. aeruginosa have been recognised from clinical and environmental sources and in Cystic Fibrosis (CF) cross infection is known to occur. There are no robust data on cross infection in NCFBr. This evidence gap impacts on managing patients but was omitted from the BTS 2010 guidelines due to the paucity of data.

Aims To seek evidence of cross infection amongst NCFBr patients.

Methods Single centre cross sectional study: We studied 50 P. aeruginosa isolates from $40 \mathrm{NCFBr}$ patients using two genotyping techniques (both blinded); an Array Tube (AT) method and Variable Number Tandem Repeat (VNTR) analysis. We included known CF clonal strains as internal controls. We then compared the data using genotype databases.

Results This is the largest cross infection study to our knowledge. We demonstrated that shared P. aeruginosa NCFBr genotypes were infrequent. Twelve patient isolates did not match any other isolate within the NCFBr collection or the databases. The most common clone, clone C $(10 \%)$, is also known to be abundant in the environment. In ten patients where longitudinal isolates were examined, paired isolates gave matching genotyping data suggesting persistent infection. There was incomplete concordance between the Array-Tube and VNTR methods (88\% agreement).

Conclusion There were no dominant Pseudomonas aeruginosa clones in NCFBr suggesting that the most prevalent mode of infection is sporadic and cross infection is rare. This may reflect the local infection control measures however. Multicentre studies are suggested to further assess the risks.

\section{Mechanisms in carcinogenesis}

\section{S127 CHEMOTHERAPY SENSITISES MALIGNANT PLEURAL MESOTHELIOMA CELLS TO UNDERGO MSC-TRAIL INDUCED APOPTOSIS}

KK Kolluri, E Sage, ZQ Yuan, A Gaingreco, SM Janes; University College London, London, United Kingdom

10.1136/thoraxjnl-2013-204457.134 